\title{
ORTHOGONAL ALMOST COMPLEX STRUCTURES ON THE RIEMANNIAN PRODUCTS OF EVEN-DIMENSIONAL ROUND SPHERES
}

\author{
Yunhee Euh and Kouei Sekigawa
}

\begin{abstract}
We discuss the integrability of orthogonal almost complex structures on Riemannian products of even-dimensional round spheres and give a partial answer to the question raised by E. Calabi concerning the existence of complex structures on a product manifold of a round 2-sphere and of a round 4-sphere.
\end{abstract}

\section{Introduction}

It is well-known that a $2 n$-dimensional sphere $S^{2 n}$ admits an almost complex structure if and only if $n=1$ or 3 and that any almost complex structure on $S^{2}$ is integrable. Also, the complex structure on $S^{2}$ is unique with respect to the conformal structure on it. A 2-dimensional sphere $S^{2}$ equipped with this complex structure is biholomorphic to a complex projective line $\mathbb{C} P_{1}$. On the contrary, it is a long-standing open problem whether $S^{6}$ admits an integrable almost complex structure (namely, a complex structure) or not. Lebrun [4] gave a partial answer to this problem, that is, proved that any orthogonal almost complex structure on a round 6-sphere is never integrable (see also [6], Corollary 5.2). On one hand, Sutherland proved that a connected product of even-dimensional spheres admits an almost complex structure if and only if it is a product of copies of $S^{2}, S^{6}$ and $S^{2} \times S^{4}$ under a more general setting ([7], Theorem 3.1). In [1], Calabi raised the problem of whether the product manifold $V^{2} \times S^{4}\left(V^{2}\right.$ is any closed orientable surface) can admit an integrable almost complex structure or not. In the present note, we discuss the integrability of orthogonal almost complex structures on a Riemannian product of round 2-spheres, 6-spheres and Riemannian product manifolds of a round 2-sphere and a round 4 -sphere, and prove the following.

Received August 8, 2011; Revised October 4, 2012.

2010 Mathematics Subject Classification. 53C15, 53C21, 53C30.

Key words and phrases. orthogonal complex structure, even-dimensional sphere, curvature identity, Ricci *-tensor.

The first author was supported by the National Research Foundation of Korea Grant funded by the Korean Government [NRF-2010-355-C00007].

(C)2013 The Korean Mathematical Society 
Theorem A. An orthogonal almost complex structure on a Riemannian product of round 2-spheres, round 6-spheres, and Riemannian product manifolds of a round 2-sphere and a round 4-sphere is integrable if and only if it is the product of the canonical complex structures on round 2-spheres.

Remark 1. Let $M$ be any Riemannian product of round 2-spheres. Then the product of the canonical complex structures of round 2-spheres is necessarily an orthogonal complex structure on $M$.

From Theorem A, we have the following partial answer to the above mentioned problem by Calabi.

Corollary B. Any orthogonal almost complex structure on a Riemannain product of a round 2-sphere and a round 4-sphere is never integrable.

Remark 2. An explicit example of an orthogonal almost Hermitian structure on a Riemannian product of a round 2-sphere and a round 4-sphere was introduced and its geometric property was discussed in [3].

We denote by $S^{m}(\kappa)$ an $m$-dimensional round sphere of positive constant sectional curvature $\kappa$. Throughout the present paper, we shall mean by a round $m$-sphere an oriented $m$-dimensional sphere with constant sectional curvature.

\section{Preliminaries}

Let $M=(M, J,\langle\rangle$,$) be a 2 n$-dimensional almost Hermitian manifold. We denote by $\nabla$ the Levi-Civita connection and $R$ the curvature tensor of $M$ defined by

$$
R(X, Y) Z=\left[\nabla_{X}, \nabla_{Y}\right]-\nabla_{[X, Y]} Z
$$

for $X, Y, Z \in \mathfrak{X}(M)$, where $\mathfrak{X}(M)$ denotes the Lie algebra of all smooth vector fields on $M$. We denote the Ricci *-tensor of $M$ by $\rho^{*}$ which is defined by

$$
\begin{aligned}
\rho^{*}(X, Y) & =\operatorname{tr}(Z \longmapsto R(X, J Z) J Y) \\
& =\frac{1}{2} \operatorname{tr}(Z \longmapsto R(X, J Y) J Z)
\end{aligned}
$$

for $X, Y, Z \in \mathfrak{X}(M)$. Here we note that the Ricci $*$-tensor $\rho^{*}$ satisfies the following equality

$$
\rho^{*}(X, Y)=\rho^{*}(J Y, J X)
$$

for $X, Y \in \mathfrak{X}(M)$. Thus from (2.3), we see that $\rho^{*}$ is symmetric if and only if $\rho^{*}$ is $J$-invariant. We also denote by $N$ the Nijenhuis tensor of the almost complex structure $J$ defined by

$$
N(X, Y)=[J X, J Y]-[X, Y]-J[J X, Y]-J[X, J Y]
$$

for $X, Y \in \mathfrak{X}(M)$. It follows from the celebrated theorem by Newlander and Nirenberg [5] that the almost complex structure $J$ is integrable if and only 
if $N=0$ holds everywhere on $M$. An almost Hermitian manifold with an integrable almost complex structure is called a Hermitian manifold.

Now we set

$$
R(X, Y, Z, W)=\langle R(X, Y) Z, W\rangle
$$

for $X, Y, Z, W \in \mathfrak{X}(M)$. Gray [2] proved the following result which plays an important role in our forthcoming arguments of the present paper.

Theorem 2.1. The curvature tensor $R$ of a Hermitian manifold $M=(M, J,\langle\rangle$, satisfies the following identity:

$$
\begin{aligned}
R_{W X Y Z} & +R_{J W J X J Y J Z}-R_{J W J X Y Z}-R_{J W X J Y Z} \\
& -R_{J W X Y J Z}-R_{W J X J Y Z}-R_{W J X Y J Z}-R_{W X J Y J Z}=0
\end{aligned}
$$

for any $W, X, Y, Z \in \mathfrak{X}(M)$.

\section{Lemmas}

We shall prove several lemmas prior to the proof of Theorem A. First of all, we note that orthogonal almost complex structures on the Riemannian products of even-dimensional round spheres do not depend on the order of the factors. We now consider the Riemannian product $M=S^{2}(\alpha) \times M^{\prime}$, where $M^{\prime}$ is a Riemannian product of round 2-spheres, round 6-spheres and Riemannian product manifolds of a round 2 -sphere and a round 4 -sphere.

Lemma 3.1. Let $J$ be an orthogonal complex structure on $M$. Then $J$ induces a canonical complex structure on $S^{2}(\alpha)$ and an orthogonal almost complex structure on $\left\{p_{1}\right\} \times M^{\prime}$ for each point $p_{1} \in S^{2}(\alpha)$.

Proof. We denote by $\pi_{1}$ and $\pi_{2}$ the canonical projections defined by $\pi_{1}: M \rightarrow$ $S^{2}(\alpha)$ and $\pi_{2}: M \rightarrow M^{\prime}$, respectively. We set

$$
x_{1}=d \pi_{1}(x), \quad x_{2}=d \pi_{2}(x)
$$

for any $x \in T_{p} M, p=\left(p_{1}, p_{2}\right) \in S^{2}(\alpha) \times M^{\prime}$. The tangent space $T_{p} M$ is identified with the orthogonal direct sum of $T_{p_{1}} S^{2}(\alpha)$ and $T_{p_{2}} M^{\prime}$ in the natural way. Let $x, y \in T_{p_{1}} S^{2}(\alpha)$ with $x \perp y,|x|=|y|=1$. Then we get

$$
R(x, y, x, y)=-\alpha .
$$

Here, since $\operatorname{dim} S^{2}(\alpha)=2$, we may set

$$
(J x)_{1}=\langle J x, y\rangle y, \quad(J y)_{1}=\langle J y, x\rangle x .
$$

Now, taking account of (3.3), we further have

$$
\begin{aligned}
& R(J x, J y, J x, J y) \\
= & R\left((J x)_{1}+(J x)_{2},(J y)_{1}+(J y)_{2},(J y)_{1}+(J y)_{2},(J x)_{1}+(J x)_{2}\right) \\
= & R\left((J x)_{1},(J y)_{1},(J x)_{1},(J y)_{1}\right)+R\left((J x)_{2},(J y)_{2},(J x)_{2},(J y)_{2}\right) \\
= & -\alpha\left(\left|(J x)_{1}\right|^{2}\left|(J y)_{1}\right|^{2}-\left\langle(J x)_{1},(J y)_{1}\right\rangle^{2}\right) \\
& +R\left((J x)_{2},(J y)_{2},(J x)_{2},(J y)_{2}\right)
\end{aligned}
$$




$$
=-\alpha\left|(J x)_{1}\right|^{2}\left|(J y)_{1}\right|^{2}+R_{2}\left((J x)_{2},(J y)_{2},(J x)_{2},(J y)_{2}\right),
$$

where $R_{2}$ is the curvature tensor of $M^{\prime}$.

$$
\begin{aligned}
R(J x, J y, x, y) & =R\left((J x)_{1},(J y)_{1}, x, y\right) \\
= & \langle J x, y\rangle\langle x, J y\rangle R(y, x, x, y) \\
= & \alpha\langle J x, y\rangle\langle x, J y\rangle \\
= & -\alpha\langle x, J y\rangle^{2}, \\
R(J x, y, J x, y) & =R\left((J x)_{1}, y,(J x)_{1}, y\right) \\
& =\langle J x, y\rangle^{2} R(y, y, y, y) \\
& =0, \\
R(J x, y, x, J y) & =R\left((J x)_{1}, y, x,(J y)_{1}\right) \\
& =-\langle J x, y\rangle^{2} R(y, y, x, x) \\
& =0, \\
R(x, J y, x, J y) & =R\left(x,(J y)_{1}, x,(J y)_{1}\right) \\
& =\langle J y, x\rangle^{2} R(x, x, x, x) \\
& =0 .
\end{aligned}
$$

Thus, from Theorem 2.1 and (3.2) (3.8), we have

$$
\begin{aligned}
0= & R(x, y, x, y)+R(J x, J y, J x, J y)-2 R(J x, J y, x, y) \\
& -R(J x, y J x, y)-2 R(J x, y, x, J y)-R(x, J y, x, J y) \\
= & -\alpha\left\{1-\left|(J x)_{1}\right|^{2}\left|(J y)_{1}\right|^{2}\right\}+R_{2}\left((J x)_{2},(J y)_{2},(J x)_{2},(J y)_{2}\right) .
\end{aligned}
$$

Since $M^{\prime}$ is non-negatively curved, we see that

$$
R_{2}\left((J x)_{2},(J y)_{2},(J x)_{2},(J y)_{2}\right) \leq 0
$$

for all $x, y \in T_{p_{1}} S^{2}(\alpha)$. Thus, from (3.9) and (3.1), we see that

$$
\left|(J x)_{1}\right|=1 \text { and }\left|(J y)_{1}\right|=1
$$

and hence $J x \in T_{p_{1}} S^{2}(\alpha)$ and $J y \in T_{p_{1}} S^{2}(\alpha)$ for any orthogonal pair $\{x, y\}$ in $T_{p_{1}} S^{2}(\alpha)$. Since $d \pi_{1}$ is a linear map from $T_{p} M$ onto $T_{p_{1}} S^{2}(\alpha)$, from (3.11), we may easily see that $J x \in T_{p_{1}} S^{2}(\alpha)$ for all $x \in T_{p_{1}} S^{2}(\alpha)$, and hence $J\left(T_{p_{1}} S^{2}(\alpha)\right)=T_{p_{1}} S^{2}(\alpha)$. Therefore we see also that $J\left(T_{p_{2}} M^{\prime}\right)=T_{p_{2}} M^{\prime}$.

Now, for each $p_{1} \in S^{2}(\alpha)$, we denote by $J^{\prime}=J^{\prime}\left(p_{1}\right)$ the induced almost complex structure on $\left\{p_{1}\right\} \times M^{\prime}$ as in Lemma 3.1. Then we have the following.

Lemma 3.2. The almost complex structure $J^{\prime}$ is integrable (and hence defines a complex structure on $\left.\left\{p_{1}\right\} \times M^{\prime}\right)$. 
Proof. Let $N^{\prime}$ be the Nijenhuis tensor of the almost complex structure $J^{\prime}$. Taking account of Lemma 3.1, we have

$$
\begin{aligned}
N^{\prime}\left(X^{\prime}, Y^{\prime}\right) & =\left[J^{\prime} X^{\prime}, J^{\prime} Y^{\prime}\right]-\left[X^{\prime}, Y^{\prime}\right]-J^{\prime}\left[J^{\prime} X^{\prime}, Y^{\prime}\right]-J^{\prime}\left[X^{\prime}, J^{\prime} Y^{\prime}\right] \\
& =\left[J X^{\prime}, J Y^{\prime}\right]-\left[X^{\prime}, Y^{\prime}\right]-J^{\prime}\left[J X^{\prime}, Y^{\prime}\right]-J^{\prime}\left[X^{\prime}, J Y^{\prime}\right] \\
& =\left[J X^{\prime}, J Y^{\prime}\right]-\left[X^{\prime}, Y^{\prime}\right]-J\left[J X^{\prime}, Y^{\prime}\right]-J\left[X^{\prime}, J Y^{\prime}\right] \\
& =N\left(X^{\prime}, Y^{\prime}\right) \\
& =0
\end{aligned}
$$

for all $X^{\prime}, Y^{\prime} \in \mathfrak{X}\left(M^{\prime}\right)$. Therefore, from (3.12), we see that the induced almost complex structure $J^{\prime}$ on $\left\{p_{1}\right\} \times M^{\prime}$ is integrable for each $p_{1} \in S^{2}(\alpha)$.

From Lemmas 3.1 and 3.2 , if $M^{\prime}$ has a round 2-sphere as a factor, by a suitable reordering of the factors, we may assume that $M$ is expressed in the form $M^{\prime}=S^{2}(\alpha) \times M^{\prime \prime}$, where $M^{\prime \prime}$ is defined similarly as $M^{\prime}$. Applying Lemma 3.2 to $M^{\prime}$, it follows that the orthogonal complex structure $J^{\prime}$ induces a complex structure on $M^{\prime \prime}$. By repeating similar operations, we may assume that $M$ is expressed in the form $M=M_{1} \times M_{2}$, where $M_{1}=S_{1}^{2}\left(\alpha_{1}\right) \times \cdots \times S_{s}^{2}\left(\alpha_{s}\right)$ $\left(0 \leq \alpha_{1} \leq \cdots \leq \alpha_{s}\right)$ and $M_{2}$ does not involve a round 2-sphere, and further that the orthogonal almost complex structure $J$ on $M$ induces a canonical orthogonal complex structure on $M_{1} \times\left\{p_{2}\right\}$ for each point $p_{2} \in M_{2}$ and an orthogonal almost complex structure on $\left\{p_{1}\right\} \times M_{2}$ for each point $p_{1} \in M_{1}$, respectively. Thus, taking account of the result due to Sutherland ([7], Theorem 3.1 ), we have the following.

Lemma 3.3. Let $M$ be a Riemannian product of round 2-spheres, round 6spheres and Riemannian product manifolds of a round 2-sphere and a round 4-sphere, and $J$ be an orthogonal complex structure on $M$. Then $M$ takes of the form $M=M^{\prime} \times M^{\prime \prime}$ (after suitable reordering of the factors), where $M^{\prime}$ (resp., $M^{\prime \prime}$ ) is a Riemannian product of round 2-spheres (resp., a Riemannian product of round 6-spheres), and further, $J$ induces a canonical orthogonal complex structure on $M^{\prime} \times\left\{p^{\prime \prime}\right\}$ for each point $p^{\prime \prime} \in M^{\prime \prime}$ and an orthogonal complex structure on $\left\{p^{\prime}\right\} \times M^{\prime \prime}$ for each point $p^{\prime} \in M^{\prime}$, respectively.

Now, we shall show the following.

Lemma 3.4. Let $M=(M,\langle\rangle$,$) be the Riemannian product of round 6$-spheres $S_{a}^{6}\left(\beta_{a}\right)=\left(S^{6},\langle,\rangle_{a}\right)\left(0<\beta_{1} \leq \beta_{2} \leq \cdots \leq \beta_{t}, a=1,2, \ldots, t\right)$, and $J$ be an orthogonal almost complex structure on $M$. Then for each point $\left(p_{1}, \ldots, p_{a-1}, p_{a+1}\right.$, $\left.\ldots, p_{t}\right) \in S_{1}^{6}\left(\beta_{1}\right) \times \cdots \times S_{a-1}^{6}\left(\beta_{a-1}\right) \times S_{a+1}^{6}\left(\beta_{a+1}\right) \times \cdots \times S_{t}^{6}\left(\beta_{t}\right), J$ induces an orthogonal almost complex structure on $\left\{\left(p_{1}, \ldots, p_{a-1}, p_{a+1}, \ldots, p_{t}\right)\right\} \times S_{a}^{6}\left(\beta_{a}\right)$.

Proof. Let $p=\left(p_{1}, p_{2}, \ldots, p_{t}\right) \in M\left(p_{a} \in S_{a}^{6}\left(\beta_{a}\right), a=1,2, \ldots, t\right)$ be any point of $M$ and $\left\{e(a)_{i}\right\}(i=1,2, \ldots, 6)$ be any orthonormal basis of $T_{p_{a}} S_{a}^{6}\left(\beta_{a}\right)$. We denote by $R_{(a)}$ the curvature tensor of $S_{a}^{6}\left(\beta_{a}\right)$. Then we have

$$
R(x, y) z=R_{(a)}(x, y) z
$$


and

$$
R_{(a)}(x, y) z=\beta_{a}\left(\langle y, z\rangle_{a} x-\langle x, z\rangle_{a} y\right)
$$

for $x, y, z \in T_{p_{a}} S_{a}^{6}\left(\beta_{a}\right)$. Now, we set

$$
J e(a)_{i}=\sum_{c=1}^{t}\left(\sum_{j=1}^{6} J(a, c)_{i j} e(c)_{j}\right)
$$

for $1 \leq i \leq 6$ and $1 \leq a \leq t$. Then since $\left\langle J e(a)_{i}, e(b)_{j}\right\rangle=-\left\langle e(a)_{i}, J e(b)_{j}\right\rangle$, from (3.15), we have

$$
\begin{aligned}
\left\langle J e(a)_{i}, e(b)_{j}\right\rangle & =\left\langle\sum_{c} \sum_{k} J(a, c)_{i k} e(c)_{k}, e(b)_{j}\right\rangle \\
& =\sum_{c} \sum_{k} J(a, c)_{i k} \delta_{c b} \delta_{k j} \\
& =J(a, b)_{i j}
\end{aligned}
$$

and

$$
\begin{aligned}
\left\langle e(a)_{i}, J e(b)_{j}\right\rangle & =\left\langle e(a)_{i}, \sum_{c} \sum_{k} J(b, c)_{j k} e(c)_{k}\right\rangle \\
& =\sum_{c} \sum_{k} J(b, c)_{j k} \delta_{a c} \delta_{i k} \\
& =J(b, a)_{j i} .
\end{aligned}
$$

Hence we have

$$
J(a, b)_{i j}=-J(b, a)_{j i}
$$

for $1 \leq a, b \leq t$ and $1 \leq i, j \leq 6$. On one hand, since $J^{2}=-i d$, from (3.15), we have

$$
\begin{aligned}
-e(a)_{i} & =J\left(J e(a)_{i}\right) \\
& =J\left(\sum_{c} \sum_{j} J(a, c)_{i j} e(c)_{j}\right) \\
& =\sum_{c} \sum_{d} \sum_{j, k} J(a, c)_{i j} J(c, d)_{j k} e(d)_{k}
\end{aligned}
$$

and hence,

$$
\sum_{c} \sum_{j} J(a, c)_{i j} J(c, d)_{j k}=-\delta_{i k} \delta_{a d}
$$

for $1 \leq i, k \leq 6$ and $1 \leq a, d \leq t$. Here, we shall calculate the components of the Ricci $*$-tensor $\rho^{*}$. From (3.13), (3.15), (3.16) and (3.17), we have

$$
\begin{aligned}
& \rho^{*}\left(e(a)_{i}, e(a)_{j}\right) \\
= & -\frac{1}{2} \sum_{c} \sum_{k} R\left(e(a)_{i}, J e(a)_{j}, e(c)_{k}, J e(c)_{k}\right)
\end{aligned}
$$




$$
\begin{aligned}
= & -\frac{1}{2} \sum_{k} R\left(e(a)_{i}, J e(a)_{j}, e(a)_{k}, J e(a)_{k}\right) \\
= & -\frac{1}{2} \sum_{k} R_{(a)}\left(e(a)_{i}, \sum_{l} J(a, a)_{j l} e(a)_{l}, e(a)_{k}, \sum_{u} J(a, a)_{k u} e(a)_{u}\right) \\
= & -\frac{1}{2} \sum_{k, l, u} J(a, a)_{j l} J(a, a)_{k u} R_{(a)}\left(e(a)_{i}, e(a)_{l}, e(a)_{k}, e(a)_{u}\right) \\
= & -\frac{\beta_{a}}{2} \sum_{k, l, u} J(a, a)_{j l} J(a, a)_{k u}\left\{\delta_{l k} \delta_{i u}-\delta_{i k} \delta_{l u}\right\} \\
= & -\frac{\beta_{a}}{2}\left\{-\delta_{j i}-\delta_{j i}\right\} \\
= & \beta_{a} \delta_{i j}, \\
& \rho^{*}\left(e(a)_{i}, e(b)_{j}\right) \\
= & -\frac{1}{2} \sum_{c} \sum_{k} R\left(e(a)_{i}, J e(b)_{j}, e(c)_{k}, J e(c)_{k}\right) \\
= & -\frac{1}{2} \sum_{k} R\left(e(a)_{i}, \sum_{l} J(b, a)_{j l} e(a)_{l}, e(a)_{k}, \sum_{u} J(a, a)_{k u} e(a)_{u}\right) \\
= & -\frac{1}{2} \sum_{k, l, u} J(b, a)_{j l} J(a, a)_{k u} R_{(a)}\left(e(a)_{i}, e(a)_{l}, e(a)_{k}, e(a)_{u}\right) \\
= & \beta_{a} \delta_{i j} \delta_{a b}, \\
= & \beta_{k, l, u} J(b, a)_{j l} J(a, a)_{k u}\left\{\delta_{l k} \delta_{i u}-\delta_{i k} \delta_{l u}\right\} \\
= & \left.\beta_{j i} \delta_{b a}-\delta_{j i} \delta_{b a}\right\} \\
= & \left.J(a, a)_{k i}-\sum_{l} J(a, a)_{i l}\right\} \\
= & \\
= & \\
= &
\end{aligned}
$$

$$
\begin{aligned}
& \rho^{*}\left(e(a)_{i}, J e(a)_{j}\right) \\
= & \frac{1}{2} \sum_{c} \sum_{k} R\left(e(a)_{i}, e(a)_{j}, e(c)_{k}, J e(c)_{k}\right) \\
= & \frac{1}{2} \sum_{k} R\left(e(a)_{i}, e(a)_{j}, e(a)_{k}, J e(a)_{k}\right) \\
= & \frac{1}{2} \sum_{k, l} J(a, a)_{k l} R_{(a)}\left(e(a)_{i}, e(a)_{j}, e(a)_{k}, e(a)_{l}\right) \\
= & \frac{\beta_{a}}{2} \sum_{k, l} J(a, a)_{k l}\left\{\delta_{j k} \delta_{i l}-\delta_{i k} \delta_{j l}\right\}
\end{aligned}
$$




$$
\begin{aligned}
= & \frac{\beta_{a}}{2}\left\{J(a, a)_{j i}-J(a, a)_{i j}\right\} \\
= & \beta_{a} J(a, a)_{j i}, \\
& \rho^{*}\left(e(a)_{i}, J e(b)_{j}\right) \\
= & \frac{1}{2} \sum_{c} \sum_{k} R\left(e(a)_{i}, e(b)_{j}, e(c)_{k}, J e(c)_{k}\right) \\
= & \frac{1}{2} \sum_{c, d} \sum_{k, l} J(c, d)_{k l} R\left(e(a)_{i}, e(b)_{j}, e(c)_{k}, e(d)_{l}\right) \\
= & -\beta_{a} \delta_{a b} J(a, b)_{i j},
\end{aligned}
$$

(3.22) $\quad \rho^{*}\left(J e(a)_{i}, e(a)_{j}\right)$

$$
\begin{aligned}
= & -\frac{1}{2} \sum_{c} \sum_{k} R\left(J e(a)_{i}, J e(a)_{j}, e(c)_{k}, J e(c)_{k}\right) \\
= & -\frac{1}{2} \sum_{c} \sum_{k, l, u, v} J(a, c)_{i l} J(a, c)_{j u} J(c, c)_{k v} R_{(c)}\left(e(c)_{l}, e(c)_{u}, e(c)_{k}, e(c)_{v}\right) \\
= & -\frac{1}{2} \sum_{c} \beta_{c} \sum_{k, l, u, v} J(a, c)_{i l} J(a, c)_{j u} J(c, c)_{k v}\left\{\delta_{u k} \delta_{l v}-\delta_{l k} \delta_{u v}\right\} \\
= & -\frac{1}{2} \sum_{c} \beta_{c}\left\{\sum_{k, l} J(a, c)_{i l} J(a, c)_{j k} J(c, c)_{k l}\right. \\
& \left.-\sum_{k, u} J(a, c)_{i k} J(a, c)_{j u} J(c, c)_{k u}\right\} \\
= & -\frac{1}{2} \sum_{c} \beta_{c}\left\{-\sum_{l} J(a, c)_{i l} \delta_{j l} \delta_{a c}+\sum_{u} J(a, c)_{j u} \delta_{i u} \delta_{a c}\right\} \\
= & \frac{1}{2} \beta_{a} J(a, a)_{i j}-\frac{1}{2} \beta_{a} J(a, a)_{j i} \\
= & \beta_{a} J(a, a)_{i j},
\end{aligned}
$$

(3.23) $\quad \rho^{*}\left(J e(b)_{i}, e(a)_{j}\right)$

$$
\begin{aligned}
& =-\frac{1}{2} \sum_{c} \sum_{k} R\left(J e(b)_{i}, J e(a)_{j}, e(c)_{k}, J e(c)_{k}\right) \\
& =-\frac{1}{2} \sum_{c} \sum_{k, l, u, v} J(b, c)_{i l} J(a, c)_{j u} J(c, c)_{k v} R_{(c)}\left(e(c)_{l}, e(c)_{u}, e(c)_{k}, e(c)_{v}\right) \\
& =-\frac{1}{2} \sum_{c} \beta_{c} \sum_{k, l, u, v} J(b, c)_{i l} J(a, c)_{j u} J(c, c)_{k v}\left\{\delta_{u k} \delta_{l v}-\delta_{l k} \delta_{u v}\right\} \\
& =-\frac{1}{2} \sum_{c} \beta_{c}\left\{\sum_{k, l} J(b, c)_{i l} J(a, c)_{j k} J(c, c)_{k l}\right.
\end{aligned}
$$




$$
\begin{aligned}
& \left.-\sum_{k, u} J(b, c)_{i k} J(a, c)_{j u} J(c, c)_{k u}\right\} \\
= & -\frac{1}{2} \sum_{c} \beta_{c}\left\{-\sum_{l} \delta_{j l} \delta_{a c} J(b, c)_{i l}+\sum_{u} \delta_{i u} \delta_{b c} J(a, c)_{j u}\right\} \\
= & \frac{1}{2} \beta_{a} J(b, a)_{i j}-\frac{1}{2} \beta_{a} J(a, b)_{j i} \\
= & -\beta_{a} J(a, b)_{i j} .
\end{aligned}
$$

Thus, from (3.18) and (3.19), we see that $\rho^{*}$ is symmetric (and hence $J$ invariant). Further, from (3.21), (3.23) and taking account of the symmetry of $\rho^{*}$, we have $J(a, b)_{i j}=0$ for $a \neq b$. Hence

$$
J\left(T_{p_{a}} S_{a}^{6}\left(\beta_{a}\right)\right)=T_{p_{a}} S_{a}^{6}\left(\beta_{a}\right), \quad a=1,2, \ldots, t .
$$

Therefore, from (3.24), we see that $J$ induces an almost complex structure on $\left\{\left(p_{1}, \ldots, p_{a-1}, p_{a+1}, \ldots, p_{t}\right)\right\} \times S_{a}^{6}\left(\beta_{a}\right)$ for each $\left(p_{1}, \ldots, p_{a-1}, p_{a+1}, \ldots, p_{t}\right) \in$ $S_{1}^{6}\left(\beta_{1}\right) \times \cdots \times S_{a-1}^{6}\left(\beta_{a-1}\right) \times S_{a+1}^{6}\left(\beta_{a+1}\right) \times \cdots \times S_{t}^{6}\left(\beta_{t}\right)$.

Lemma 3.5. Any orthogonal almost complex structure on a Riemannian product of round 6-spheres is never integrable.

Proof. Let $M=(M,\langle\rangle$,$) be a Riemannian product of round 6-spheres S_{a}^{6}\left(\beta_{a}\right)$ $(a=1,2, \ldots, t)$ and assume that $M$ admits an orthogonal complex structure denoted by $J$. Then taking account of the results in [4], it suffices to consider the case when $t \geq 2$. From Lemma 3.4, for each point $\left(p_{1}, \ldots, p_{t-1}\right) \in$ $S_{1}^{6}\left(\beta_{1}\right) \times \cdots \times S_{t-1}^{6}\left(\beta_{t-1}\right), J$ induces an orthogonal almost complex structure on $\left\{\left(p_{1}, \ldots, p_{t-1}\right)\right\} \times S_{t}^{6}\left(\beta_{t}\right)$. Then we may show that the induced orthogonal almost complex structure is integrable by slightly modifying the proofs of Lemmas 3.1 and 3.2. But this is a contradiction.

\section{Proof of Theorem A}

In this section, we prove Theorem A based on the arguments in $\S 3$. Let $M=(M,\langle\rangle$,$) be a Riemannian product of round 2-spheres, round 6-spheres,$ and Riemannian product manifolds of a round 2-sphere and a round 4-sphere. Assume that $M$ admits an orthogonal complex structure and denote it by $J$. Then from Lemma 3.3, we see that $M$ is of the form $M=M^{\prime} \times M^{\prime \prime}$, where $M^{\prime}$ is of the form $M^{\prime}=S^{2}\left(\alpha_{1}\right) \times \cdots \times S^{2}\left(\alpha_{s}\right)$ and $M^{\prime \prime}$ is of the form $M^{\prime \prime}=$ $S^{6}\left(\beta_{1}\right) \times \cdots \times S^{6}\left(\beta_{t}\right)$, respectively. Further, $J$ induces an orthogonal complex structure on $\left\{p^{\prime}\right\} \times M^{\prime \prime}$ for each point $p^{\prime} \in M^{\prime}$. Therefore, from Lemmas 3.1 and 3.2 and by the uniqueness of the canonical complex structure on a round 2sphere, $J$ is an orthogonal complex structure on $M$. Therefore, taking account of Lemma 3.1, we see that $J$ is a product of the canonical complex structures on these round 2-spheres. The converse is evident by Remark 1 . This completes the proof of Theorem A. 
Acknowledgements. The authors would like to express their thanks to Professor H. Hashimoto for drawing their attention to the present topic of this paper and also to the referee for his valuable suggestions.

\section{References}

[1] E. Calabi, Construction and properties of some 6-dimensional almost complex manifold, Trans. Amer. Math. Soc. 87 (1958), 407-438.

[2] A. Gray, Curvature identities for Hermitian and almost Hermitian manifolds, Tôhoku Math. J. 28 (1976), no. 4, 601-612.

[3] H. Hashimoto, T. Koda, K. Mashimi, and K. Sekigawa, Extrinsic homogeneous almost Hermitian 6-dimensional submanifolds in the Octonions, Kodai Math. J. 30 (2007), no. 3, 297-321.

[4] C. Lebrun, Orthogonal complex structures on $S^{6}$, Proc. Amer. Math. Soc. 101 (1987), no. $1,136-138$.

[5] A. Newlander and L. Nirenberg, Complex analytic coordinates in almost complex manifolds, Ann. of Math. 65 (1957), 391-404.

[6] K. Sekigawa and L. Vanhecke, Almost Hermitian manifolds with vanishing first Chern class or Chern numbers, Rend. Sem. Mat. Univ. Politec. Torino 50 (1992), no 2, 195-208.

[7] W. A. Sutherland, A note on almost complex and weakly complex structures, J. London Math. Soc. 40 (1965), 705-712.

YUNHEE EUH

Department of Mathematics

SUNGKYUNKWAN UNIVERSITY

SuWON 440-746, KoreA

E-mail address: prettyfish@skku.edu

Kouei Sekigawa

Department of Mathematics

FACUlTy OF SCIENCE

NiIgAta University

NiIgATA 950-2181, JAPAN

E-mail address: sekigawa@math.sc.niigata-u.ac.jp 